Tôhoku Math. Journ.

29 (1977), 125-136.

\title{
SOME SYSTEM OF DIFFERENTIAL EQUATIONS \\ ON RIEMANNIAN MANIFOLDS AND ITS \\ APPLICATIONS TO CONTACT STRUCTURES
}

\author{
ShÛKICHI TANNo
}

(Received January 26, 1976)

1. Introduction. Let $(M, g)$ be a connected $m(\geq 2)$-dimensional Riemannian manifold of class $C^{\infty}$. The existence of a function satisfying some differential equations on $(M, g)$ sometimes determines differential geometric properties of $(M, g)$. For example, a complete $(M, g)$ admits a non-trivial (global) solution $f$ of the system of differential equations

$$
\nabla_{j} \nabla_{i} f+K f g_{j i}=0
$$

for some positive constant $K$, if and only if $(M, g)$ is isometric to a Euclidean sphere $S^{m}(K)$ of radius $1 / \sqrt{K}$ (S. Ishihara and Y. Tashiro [7], M. Obata [11], [12]).

Furthermore, M. Obata [12] announced the following.

OBATA's THEOREM (*). Let $(M, g)$ be a complete and simply connected Riemannian manifold of dimension $m$. In order for $M$ to admit a nontrivial solution $f$ for the system of differential equations

$$
\nabla_{j} \nabla_{i} \nabla_{h} f+K\left(2 \nabla_{j} f g_{i h}+\nabla_{i} f g_{h j}+\nabla_{h} f g_{j i}\right)=0
$$

for some positive constant $K$, it is necessary and sufficient that $(M, g)$ be isometric to a Euclidean sphere $S^{m}(K)$.

This system of differential equations is of order 3 , and this result is itself of great importance. However, the complete proof has not yet been published unfortunately.

On the other hand, a unit Killing vector field $\xi$ on $(M, g)$ is called a normal contact structure or simply a Sasakian structure, if it satisfies

$$
R(X, \xi) Y=g(\xi, Y) X-g(X, Y) \xi
$$

for any vector fields (or tangent vectors) $X$ and $Y$, where $R$ denotes the Riemannian curvature tensor field. In other words, $\xi$ is a Sasakian structure if and only if it belongs to the 1-nullity distribution of $(M, g)$. In this case, the dimension $m$ of $M$ is odd.

Let $\xi$ and $\eta$ be two Sasakian structures on $(M, g)$. If $g(\xi, \eta)=f$ is not constant, then $f$ satisfies (1.2) for $K=1$ (S. Tachibana and W. N. 
Yu [17]). Applying Theorem (*), S. Tachibana and W. N. Yu [17] showed the following.

THEOREM (**). Let $(M, g)$ be a complete and simply connected Riemannian manifold. If $(M, g)$ admits two Sasakian structures $\xi$ and $\eta$ with non-constant $g(\xi, \eta)$, then $(M, g)$ is isometric to $S^{m}(1)$.

In this paper, without assuming Theorem $(*)$ we give a proof of Theorem $(* *)$ for the dimension $m \leq 11$. For this purpose, first we prove Theorem $\mathrm{A}$ as a weaker version of Theorem $(*)$, and then we apply a Theorem by D. Ferus [4] on 1-nullity distributions.

THEOREM A. Let $(M, g)$ be a complete Riemannian manifold. If $(M, g)$ admits a non-trivial function $f$ satisfying (1.2) and

$$
(1 / K) g^{h j} \nabla_{h} f \nabla_{j} \nabla_{i} f=-4 f \nabla_{i} f,
$$

then $(M, g)$ is of constant curvature $K$.

This is a special case of Theorem (*) as will be explained in the Section 4. However, our proof is differential-geometric and we can give a nice frame at a point concretely, which plays an important rôle in the outline of Obata's proof of Theorem $(*)$.

2. Preliminaries. Let $(M, g)$ be a Riemannian manifold with a normal contact structure or a Sasakian structure $\xi$ (cf. S. Sasaki [15], S. Tanno [18], [19]). Since $\xi$ is a Killing vector field, (1.3) is written as

$$
\nabla_{X}(\nabla \xi) \cdot Y=g(\xi, Y) X-g(X, Y) \xi,
$$

where $X$ and $Y$ are vector fields or tangent vectors. If we put $\nabla \xi=-\phi$ (i.e., $\left.\nabla_{X} \xi=-\phi X\right)$, then the $(1,1)$-tensor field $\phi$ satisfies the following.

$$
\begin{gathered}
\phi \xi=0, \quad \phi \phi X=-X+g(\xi, X) \xi, \\
g(\phi X, \phi Y)=g(X, Y)-g(\xi, X) g(\xi, Y), \\
\nabla_{j} \phi_{i}^{h}=g_{j i} \xi^{h}-\delta_{j}^{h} \xi_{i} .
\end{gathered}
$$

The 1-form which is dual to $\xi$ with respect to $g$ is called a contact form on $M$.

We pick up some known results for our later arguments.

[i] If $\phi$ is an isometry of $(M, g)$ and $\xi$ is a Sasakian structure on $(M, g)$, then $\phi \xi$ is also a Sasakian structure on $(M, g)$, where $\varphi$ denotes also its differential (S. Tanno [19]).

[ii] If $\xi$ and $\eta$ are Sasakian structures on $(M, g)$, then $g(\xi, \eta)=f$ satisfies (1.2) for $K=1$ (S. Tachibana and W. N. Yu [17]).

[iii] If $\xi$ and $\eta$ are two Sasakian structures on $(M, g)$ and if $g(\xi, \eta)=f$ 
is constant $(\neq 1, \neq-1)$, then putting $\xi_{(1)}=\xi$,

$$
\xi_{(2)}=(\eta-f \xi) /|\eta-f \xi|,
$$

and $\xi_{(3)}=\left[\xi_{(1)}, \xi_{(2)}\right] / 2$, we have a Sasakian 3-structure $\left(\xi_{(1)}, \xi_{(2)}, \xi_{(3)}\right)$ (cf. Y. Y. Kuo [9]). A Riemannian manifold admitting a Sasakian 3-structure is an Einstein space (T. Kashiwada [8]).

The Riemannian curvature tensor field $R$ is defined by

$$
R(X, Y) Z=\nabla_{X} \nabla_{Y} Z-\nabla_{Y} \nabla_{X} Z-\nabla_{[X, Y]} Z \text {. }
$$

We define a curvature-like tensor field $T$ by

$$
T(X, Y) Z=R(X, Y) Z-K(g(Y, Z) X-g(X, Z) Y) .
$$

Let $x$ be a point of $M$ and let $M_{x}$ be the tangent space to $M$ at $x$. Then the $K$-nullity space $N_{x}$ at $x$ is defined by

$$
N_{x}=\left\{X \in M_{x} ; T(X, Y) Z=0 \text { for all } Y, Z \in M_{x}\right\} .
$$

$\operatorname{dim} N_{x}=\mu(x)$ is called the index of $K$-nullity at $x$ (S. S. Chern and N. H. Kuiper [2], T. Otsuki [13]). The minimum $\mu$ of $\mu(x)$ on $M$ is called the index of $K$-nullity of $(M, g)$.

[iv] $\mu(x)$ is upper semi-continuous, and hence the set $G$ of $M$ where $\mu(x)=\mu$ is open.

[v] If $\mu(x)$ is constant on an open submanifold $M^{0}$ of $M$, then the $K$-nullity distribution: $x \rightarrow N_{x}$ is integrable on $M^{0}$, and leaves (maximal integral submanifolds) are totally geodesic. Therefore leaves are of constant curvature (K. Abe [1], A. Gray [6], R. Maltz [10], etc.).

[vi] If $(M, g)$ is complete and if $\mu \geq 1$, then leaves on $G$ are complete (K. Abe [1], Y. N. Clifton and R. Maltz [3], D. Ferus [5], etc.).

[vii] Let $N$ be a $\mu$-dimensional involutive (integrable) distribution. Assume that:

(a) each leaf of $N$ is complete and totally geodesic,

(b) sectional curvature $K(X, Y)=$ constant $>0$ for $X \in N, Y \in N^{\perp}$, where $N^{\perp}$ denotes the distribution orthocomplementary to $N$ (in case $\mu<m$ ),

(c) $\mu>\nu_{m}$, where $\nu_{m}$ is for example;

$$
\begin{aligned}
& m \text { : } 234 \quad 567 \quad 8910 \quad 1112 \quad 13141516 \quad 171819 \cdots 24252627 \ldots
\end{aligned}
$$

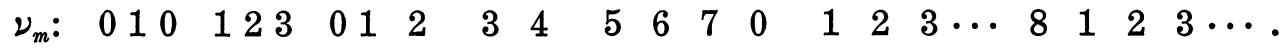

Then we have $\mu=m$ (D. Ferus [4]).

Next we summerise the fundamental facts on (1.2).

[viii] If a function $f$ on $(M, g)$ satisfies (1.2), then $\left(\nabla^{i} f\right)=\left(g^{i j} \nabla_{j} f\right)$ is an infinitesimal projective transformation. This follows from (1.2) and the Ricci identity for $\nabla_{j} \nabla_{i} \nabla_{h} f-\nabla_{i} \nabla_{j} \nabla_{h} f$ (M. Obata [12]). 
[ix] Let $\{x(s)\}$ be a complete geodesic in $(M, g)$ with arclength parameter $s$. Then the restriction of $f$ satisfying (1.2) with $K=1$ to $\{x(s)\}$ is given by

$$
f(s)=\left(f^{\prime \prime}(0) / 2\right) \sin ^{2} s+\left(f^{\prime}(0) / 2\right) \sin 2 s+f(0) .
$$

This is verified by the fact that (1.2) on the geodesic is $f^{\prime \prime \prime}+4 f^{\prime}=0$, where the dash denotes $d / d s$. In particular, $f(s)$ is bounded and periodic $(f(\pi+s)=f(s))$. Furthermore, if $f\left(s_{0}\right)$ is the maximum, then $f\left(s_{0}+\pi / 2\right)$ is the minimum.

[x] If $f$ satisfies (1.2), then $\left(\nabla^{i} f\right)$ belongs to the $K$-nullity distribution. This follows from the Ricci identity.

3. Proof of Theorem A. By replacing $g$ by $K g$, we can assume that $K=1$ in proving Theorem A. Let $f$ be a non-trivial function on $(M, g)$ satisfying

$$
\begin{gathered}
\nabla_{j} \nabla_{i} \nabla_{h} f+2 \nabla_{j} f g_{i h}+\nabla_{i} f g_{h j}+\nabla_{h} f g_{j i}=0, \\
\nabla_{j} \nabla_{i} f \nabla^{j} f=-4 f \nabla_{i} f,
\end{gathered}
$$

where $\nabla^{j} f=g^{j h} \nabla_{h} f$, and $\nabla$ denotes the Riemannian connection of $(M, g)$. We define a vector field $F$ by $F=\operatorname{grad} f=\left(\nabla^{i} f\right)$. By (3.2) we have $\nabla_{F} F=-4 f F$, that is, each trajectory of $F$ is a geodesic. Let $\varphi_{t}=\exp t F$ be the (local) 1-parameter group of (local) transformations generated by $F$. We take and fix a trajectory $C$ of $F$ and take a point $p$ of $C$. We parameterise $C$ so that

$$
C=\{x(t)\}=\{\exp t F \cdot p\}=\left\{\varphi_{t} p\right\} .
$$

Let $f=f(t)$ be the restriction of $f$ to $C$. Then

$$
f^{*}(t)=\frac{d f}{d t}=F^{i} \frac{\partial f}{\partial x^{i}}=\nabla^{i} f \nabla_{i} f .
$$

(3.2) is written as

$$
\nabla_{i}\left(\nabla_{j} f \nabla^{j} f\right)=-4 \nabla_{i} f^{2} .
$$

Transvecting the last equation by $\nabla^{i} f$, we get

$$
f^{* *}=-4\left(f^{2}\right)^{*},
$$

where ${ }^{* *}$ denotes $d^{2} / d t^{2}$. Solving this we have

$$
f^{*}=-4 f^{2}+c
$$

for some constant $c$. If $c=0$, then

$$
f(t)=(4 t+\beta)^{-1}
$$


for some constant $\beta$. If $c<0$, then

$$
f(t)=-b \tan 4 b(t+\beta), \quad b=(-c / 4)^{1 / 2} .
$$

And if $c>0$, then

$$
f(t)=a\left(e^{8 a t+\beta}-1\right) /\left(e^{8 a t+\beta}+1\right), \quad 4 a^{2}=c,
$$

or

$$
f(t)=a\left(e^{8 a t+\beta}+1\right) /\left(e^{8 a t+\beta}-1\right), \quad 4 a^{2}=c .
$$

Since $C$ is a geodesic and $(M, g)$ is complete, we see that the parameter $t$ can be extended as far as $f^{*} \neq 0$. Therefore (3.4), (3.5) and (3.7) can not be bounded. Hence, by [ix] of $\S 2$ we get (3.6). This shows that $f(t)$ takes the value 0 once. So we assume that $f(p)=0$.

LemMa 3.1. A non-trivial trajectory $C$ of $F$ is parameterised so that $C=\{\exp t F \cdot p,-\infty<t<\infty\}, f(p)=f(0)=0$, and

$$
\begin{gathered}
f^{*}(t)=-4 f^{2}+4 a^{2}, \\
f(t)=a\left(e^{8 a t}-1\right) /\left(e^{8 a t}+1\right)
\end{gathered}
$$

for some positive constant a. In particular, $-a<f(t)<a$ and $f(t) \rightarrow a$ (as $t \rightarrow \infty$ ), $f(t) \rightarrow-a$ (as $t \rightarrow-\infty$ ).

Proof. This follows from (3.6) and $f(p)=0$. q.e.d.

Lemma 3.2. The tensor field $\left(\nabla_{j} F^{i}\right)=\left(\nabla_{j} \nabla^{i} f\right)$ is symmetric with respect to $g$ and has real eigenvalues at each point of $C$. There are at most three different eigenvalues at each point:

$$
-4 f,-2 f+2 a,-2 f-2 a,
$$

where the multiplicity of $-4 f$ is one.

Proof. $F$ is an eigenvector at each point by (3.2) for the eigenvalue $-4 f$. Let $Z$ be a (local) field of eigenvectors along $C$ which is orthogonal to $F$. We put

$$
Z^{j} \nabla_{j} \nabla^{i} f=\lambda Z^{i}
$$

Operating $\nabla_{h}$ to (3.2) we obtain

$$
\nabla_{h} \nabla_{j} \nabla_{i} f \nabla^{j} f+\nabla_{j} \nabla_{i} f \nabla_{h} \nabla^{j} f=-4 \nabla_{h} f \nabla_{i} f-4 f \nabla_{h} \nabla_{i} f .
$$

By (3.1) we have

$$
4 f \nabla_{h} \nabla_{i} f=-\nabla_{j} \nabla_{i} f \nabla_{h} \nabla^{j} f-\nabla_{h} f \nabla_{i} f+\left(\nabla_{r} f \nabla^{r} f\right) g_{i h} .
$$

Transvecting the last equation with $Z^{h} Z^{i}$ and using (3.10), we get

$$
\lambda^{2}+4 f \lambda-\left(\nabla_{r} f \nabla^{r} f\right)=0 .
$$


Hence, $\lambda$ can take at most two values. Since $\left(\nabla_{r} f \nabla^{r} f\right)=f^{*}$, applying (3.8) we can solve $\lambda$ from (3.11) and get $\lambda=-2 f \pm 2 a$.

q.e.d.

Lemma 3.3. By $L_{F}$ we denote the Lie derivation by $F$. Then

$$
L_{F}\left(\nabla_{j} \nabla^{i} f\right)=-2\left(\nabla_{r} f \nabla^{r} f\right) \delta_{j}^{i}-2 \nabla^{i} f \nabla_{j} f .
$$

Proof. By definition of $L_{F}$ we have

$$
L_{F}\left(\nabla_{j} \nabla^{i} f\right)=F^{h} \nabla_{h} \nabla_{j} \nabla^{i} f-\nabla_{h} F^{i} \nabla_{j} \nabla^{h} f+\nabla_{j} F^{h} \nabla_{h} \nabla^{i} f \text {. }
$$

Applying (3.1) we get the required relation.

q.e.d.

LEMMA 3.4. Let $D_{1}, D_{2}, D_{3}$ be the fields of subspaces along $C$ defined by the eigenspaces for $-4 f,-2 f+2 a,-2 f-2 a$, respectively. Then $D_{1}, D_{2}, D_{3}$ are orthogonal and invariant by $\varphi_{t}=\exp t F$.

Proof. It is clear that $D_{1}, D_{2}, D_{3}$ are orthogonal. Since $L_{F} F=[F, F]=0$, we have $\varphi_{t} D_{1}=D_{1}$. Next we show that $D_{2}+D_{3}$ is invariant by $\varphi_{t}$. Let $Z_{p}$ be a tangent vector at $p$ such that $Z_{p} \in\left(D_{2}+D_{3}\right)(p)$. Define a vector field $Z$ along $C$ by

$$
Z_{\varphi_{t} p}=\varphi_{t} Z_{p}
$$

where $\varphi_{t}$ denotes also its differential. Define a function $G(t)$ of $t$ by $G(t)=g_{\varphi_{t} p}(Z, F)$. Since $L_{F} Z=0, L_{F} F=0$ and $L_{F} g=\left(2 \nabla_{i} \nabla_{j} f\right)$, we get

$$
\begin{aligned}
G^{*}(t) & =\frac{d G(t)}{d t}=L_{F} G(t) \\
& =2 \nabla_{i} \nabla_{j} f Z^{i} \nabla^{j} f=-8 f G(t)
\end{aligned}
$$

by (3.2). Since actually $G(0)=0$, we have $G(t)=0$ and $Z \in D_{2}+D_{3}$. Next we put

$$
Z=Z_{2}+Z_{3}
$$

where $Z_{2}$ and $Z_{3}$ are vector fields along $C$ such that $Z_{2} \in D_{2}$ and $Z_{3} \in D_{3}$. It is easily verified that

$$
Z_{2}=\left(\nabla_{Z} F+(2 f+2 a) Z\right) / 4 a,
$$

from which the differentiability of $Z_{2}$ on $t$ follows. Operating $L_{F}$ to the both sides of the last equation, applying Lemma 3.3, and using (3.8) and $L_{F} Z=0$, we obtain

$$
L_{F} Z_{2}=0 \text {. }
$$

By (3.12) $Z_{3}$ is also differentiable and hence we get $L_{F} Z_{3}=0$. Hence, $D_{2}$ and $D_{3}$ are invariant by $\varphi_{t}$. q.e.d.

Let $\left\{\left(E_{i}\right)_{p}, i=1, \cdots, m\right\}$ be an orthonormal frame at $p$ such that 


$$
\begin{aligned}
& \left(E_{1}\right)_{p} \in D_{1}(p), \\
& \left(E_{u}\right)_{p} \in D_{2}(p), \quad u=2, \cdots, r, \\
& \left(E_{v}\right)_{p} \in D_{3}(p), \quad v=r+1, \cdots, m .
\end{aligned}
$$

We define a field of frames $\left\{E_{i}\right\}$ along $C$ by $\left(E_{i}\right)_{\varphi_{t}}=\varphi_{t}\left(E_{i}\right)_{p}$. Then $\left\{E_{u}\right\}$ is a field of basis of $D_{2}$ and $\left\{E_{v}\right\}$ is a field of basis of $D_{3}$.

LEMMA 3.5. With respect to the length of $E_{i}$, we have

$$
\begin{array}{ll}
G_{2}(t)=g_{\varphi_{t} p}\left(E_{u}, E_{u}\right)=2 e^{4 a t} /\left(e^{4 a t}+e^{-4 a t}\right), & E_{u} \in D_{2}, \\
G_{3}(t)=g_{\varphi_{t} p}\left(E_{v}, E_{v}\right)=2 e^{-4 a t} /\left(e^{4 a t}+e^{-4 a t}\right), & E_{v} \in D_{3} .
\end{array}
$$

In particular, $G_{2}(t) \rightarrow 2$ (as $t \rightarrow \infty$ ) and $G_{2}(t) \rightarrow 0$ (as $\left.t \rightarrow-\infty\right)$, while $G_{3}(t) \rightarrow 0($ as $t \rightarrow \infty)$ and $G_{3}(t) \rightarrow 2($ as $t \rightarrow-\infty)$.

Proof. Let $E_{u} \in D_{2}$. Then

$$
\begin{aligned}
G_{2}^{*}(t) & =L_{F} G_{2}(t)=2 \nabla_{j} \nabla_{i} f E_{u}^{j} E_{u}^{i} \\
& =2(2 a-2 f) G_{2}(t) .
\end{aligned}
$$

Consequently

$$
\log G_{2}(t)=\int 4(a-f) d t+L
$$

for some constant $L$. By (3.9), we get

$$
\int 4(a-f) d t=4 a t-\log \left(e^{4 a t}+e^{-4 a t}\right),
$$

and hence,

$$
G_{2}(t)=e^{L} e^{4 a t} /\left(e^{4 a t}+e^{-4 a t}\right) .
$$

Since $G_{2}(0)=1$, we get $e^{L}=2 . G_{3}(t)$ is similarly obtained.

q.e.d.

Similarly we have $g_{\varphi_{t} p}\left(E_{i}, E_{j}\right)=0$ for $i \neq j$. Thus, $\left\{E_{i}\right\}$ is a field of orthogonal frames.

Proof of Theorem A. Now we can apply Obata's method. Since the field of orthogonal frames $\left\{E_{i}\right\}$ is invariant by $\varphi_{t}$, its dual $\left\{w^{i}\right\}$ is also invariant by $\varphi_{t}$. We put

$$
{ }^{*} E_{i}=E_{i}|| E_{i}\left|, \quad{ }^{*} w^{i}=\right| E_{i} \mid w^{i} .
$$

Since the projective curvature tensor field $P$ is invariant by $\varphi_{t}$ (cf. [viii] of $\S 2), P\left(E_{i}, E_{j}, E_{k}, w^{l}\right)$ is constant on $C$. By Lemma 3.1 and $\left|E_{1}\right|(0)=1$, we obtain

$$
\left|E_{1}\right|^{2}=f^{*} / 4 a^{2}=4 /\left(e^{8 a t}+e^{-8 a t}+2\right) .
$$

Since the length of $C$ is $\pi / 2$ ([ix] of $\S 2$ ), $P\left({ }^{*} E_{i},{ }^{*} E_{j},{ }^{*} E_{k},{ }^{*} w^{l}\right.$ ) is bounded 
on $C$. On the other hand,

$$
P\left({ }^{*} E_{i},{ }^{*} E_{j},{ }^{*} E_{k},{ }^{*} w^{l}\right)=\frac{\left|E_{l}\right|}{\left|E_{i}\right|\left|E_{j}\right|\left|E_{k}\right|} P\left(E_{i}, E_{j}, E_{k}, w^{l}\right)
$$

holds on $C$. If $P\left(E_{i}, E_{j}, E_{k}, w^{l}\right) \neq 0$ for some $(i, j, k, l)$, then by Lemma 3.5 and (3.13) the right hand side can not be bounded when $t \rightarrow \infty$ or $t \rightarrow-\infty$. This is a contradiction. Thus, $P=0$ and $(M, g)$ is of constant curvature $K_{1}$. Since $\left(\nabla^{i} f\right)$ belongs to the 1-nullity distribution, $K_{1}=1$.

q.e.d.

4. Remarks. (1) As M. Obata explained in [12], the standard model of $f$ satisfying (1.2) on $S^{m}(1)$ is

$$
f=\sum a_{\alpha}\left(y^{\alpha}\right)^{2}+b,
$$

where $a_{\alpha}, b$ are constant $\left(a_{0} \geq a_{1} \geqq \cdots \geq a_{m}\right)$, and $\left(y^{\alpha} ; \alpha=0,1, \cdots, m\right)$ is the standard coordinate system of an $(m+1)$-dimensional Euclidean space $E^{m+1}$. In this case, at a critical point $\left(y^{0}=1\right),\left(\nabla_{i} \nabla^{h} f\right)$ has eigenvalues $2\left(a_{i}-a_{0}\right)$.

Proposition 4.1. A function $f$ of the form (4.1) satisfies (1.4) for $K=1$, if and only if

$$
\begin{aligned}
f= & a\left(y^{0}\right)^{2}+\cdots+a\left(y^{r}\right)^{2} \\
& +(a+h)\left(y^{r+1}\right)^{2}+\cdots+(a+h)\left(y^{m}\right)^{2}-a-h / 2,
\end{aligned}
$$

where $a$ and $h$ are constant and $1 \leq r \leq m-1$.

Proof. Let $\left(U, x^{i}\right)$ be a local coordinate neighborhood such that $x^{i}=y^{i}(i=1, \cdots, m), y^{0}=[1-\Delta]^{1 / 2}\left(\Delta=\sum\left(x^{i}\right)^{2}\right), y^{0}>0$. Then we have

$$
\begin{aligned}
g_{i j} & =\delta_{i j}+x^{i} x^{j} /(1-\Delta), \\
g^{j k} & =\delta^{j k}-x^{j} x^{k}, \\
\Gamma_{j k}^{i} & =x^{i} \delta_{j k}+x^{i} x^{j} x^{k} /(1-\Delta) .
\end{aligned}
$$

Since $f=\sum\left(a_{i}-a_{0}\right)\left(x^{i}\right)^{2}+a_{0}+b$, calculating (1.4) we get $\left(a_{i}-a_{0}\right)^{2} x^{i}=$ $-2\left(a_{0}+b\right)\left(a_{i}-a_{0}\right) x^{i}$, and hence (4.2). The converse is now clear.

q.e.d.

Hence, our Theorem $\mathrm{A}$ is modeled on functions (4.2).

For a function $f$ of the form (4.1), it is also verified that if each trajectory of $\operatorname{grad} f$ is a geodesic, then it is of the form (4.2) up to an additive constant.

(2) Next we explain about Sasakian structures, for simplicity, on a 3-dimensional sphere $S^{3}(1)$. We identify $S^{3}(1)$ with the space of unit quaternions $Q_{0}$. Let $i, j, k$ be units such that $i^{2}=-1, i j=-j i=k$, 
etc. For a point $q \in Q_{0}$, we define two curves

$$
\begin{aligned}
& a(t)=\cos t \boldsymbol{q}+\sin t \boldsymbol{q} \boldsymbol{i}, \\
& b(t)=\cos t \boldsymbol{q}+\sin t \boldsymbol{i q} .
\end{aligned}
$$

We define $\xi_{1}$ by $\left(\xi_{1}\right)_{q}=(d a(t) / d t)_{t=0}$ and $\eta_{1}$ by $\left(\eta_{1}\right)_{q}=(d b(t) / d t)_{t=0}$. Then $\xi_{1}$ and $\eta_{1}$ are Sasakian structures. Similarly for $j$ and $k$, we have $\xi_{2}, \eta_{2}$, $\xi_{3}, \eta_{3}$. $\left(\xi_{1}, \xi_{2}, \xi_{3}\right)$ defines a Sasakian 3-structure. By our definition, the actions of $\exp t \xi_{1}$ and $\exp s \eta_{1}$ are commutative. Hence $\left[\xi_{1}, \eta_{1}\right]=0$. Similarly $\left[\xi_{\alpha}, \eta_{\beta}\right]=0, \alpha, \beta=1, \cdots, 3$.

$\xi_{1}, \xi_{2}, \xi_{3}, \eta_{1}, \eta_{2}, \eta_{3}$ form a basis of the Lie algebra of Killing vector fields on $S^{3}(1)=Q_{0}$.

$\xi_{1}, \eta_{1}, \eta_{2}, \eta_{3}$ form a basis of the Lie algebra of infinitesimal automorphisms of the Sasakian structure $\xi_{1}$ on $S^{3}(1)$.

$g\left(\xi_{1}, \eta_{1}\right)$ is not constant and satisfies (1.2) and (1.4) (cf. Lemma 5.3).

(3) If $(M, g)$ is complete and real analytic, a paper by A. S. Sodovnikov [16] is remarkable.

\section{Applications to Sasakian structures.}

THEOREM 5.1. Let $(M, g)$ be a 3-dimensional Riemannian manifold. If $\xi$ and $\eta$ are Sasakian structures on $(M, g)$ with $\xi \neq \eta$ and $\xi \neq-\eta$, then $(M, g)$ is of constant curvature 1.

PRoof. Except for a set of measure zero, we have a 2-plane field defined by $\xi$ and $\eta$. Therefore, 1-nullity index is equal to 2 or greater than 2 , and hence $(M, g)$ is of constant curvature 1 .

q.e.d.

LEMMA 5.2. Let $\xi$ and $\eta$ be two Sasakian structures on $(M, g)$. If we put $\nabla \xi=-\phi$ and $\nabla \eta=-\psi$, then $f=g(\xi, \eta)$ satisfies

$$
\nabla^{j} f \nabla_{j} \nabla^{i} f=-3 f \nabla^{i} f-\psi_{j}^{i} \phi_{r}^{j} \psi_{s}^{r} \xi^{s}-\phi_{j}^{i} \psi_{r}^{j} \phi_{s}^{r} \eta^{s} .
$$

Proof. First we have

$$
\nabla_{i} f=\nabla_{i}\left(\xi^{r} \eta_{r}\right)=\psi_{i r} \xi^{r}+\phi_{i r} \eta^{r},
$$

where $\psi_{i r}=g_{i s} \psi_{r}^{s}$, etc. Then

$$
\begin{aligned}
\nabla_{j} \nabla_{i} f & =-\nabla_{j} \psi_{i}^{r} \xi_{r}-\psi_{i r} \phi_{j}^{r}-\phi_{i r} \psi_{j}^{r}-\eta^{r} \nabla_{j} \phi_{r i} \\
& =-2 f g_{i j}+\eta_{i} \xi_{j}+\xi_{i} \eta_{j}-\psi_{i r} \phi_{j}^{r}-\phi_{i r} \psi_{j}^{r} .
\end{aligned}
$$

By these two relations we get

$$
\nabla^{j} f \nabla_{j} \nabla_{i} f=-3 f\left(\psi_{i r} \xi^{r}+\phi_{i r} \eta^{r}\right)-\psi_{i r} \phi_{j}^{r} \psi_{s}^{j} \xi^{s}-\phi_{i r} \psi_{j}^{r} \phi_{s}^{j} \eta^{s},
$$

from which we have (5.1).

Lemma 5.3. In Lemma 5.2, if $[\xi, \eta]=0$, then 


$$
\nabla^{j} f \nabla_{j} \nabla^{i} f=-4 f \nabla^{i} f .
$$

Proof. $[\xi, \eta]=\nabla_{\xi} \eta-\nabla_{\eta} \xi=0$ implies $\phi_{r}^{i} \eta^{r}=\psi_{s}^{i} \xi^{s}$. Then, by (5.1) and (5.2), we obtain (5.3).

THEOREM 5.4. Let $(M, g)$ be a complete Riemannian manifold. If $(M, g)$ admits two Sasakian structures $\xi$ and $\eta$ with non-constant $g(\xi, \eta)$ and satisfying $[\xi, \eta]=0$, then $(M, g)$ is of constant curvature 1 .

Proof. If $[\xi, \eta]=0$, by Lemma 5.3 we have (1.4) for $K=1$. By [ii] of $\S 2, f=g(\xi, \eta)$ satisfies (1.2) for $K=1$. Therefore, Theorem A shows that $(M, g)$ is of constant curvature 1 .

THEOREM 5.5. Let $(M, g)$ be a complete Riemannian manifold of dimension $2 n+1=m ; 5 \leq m \leq 11$ (or more generally, $m=5,7,9,11$, $17,19,25,27,33, \cdots)$. If $(M, g)$ admits two Sasakian structures $\xi$ and $\eta$ with non-constant $g(\xi, \eta)$, then $(M, g)$ is of constant curvature 1.

Proof. If $[\xi, \eta]=0$, Theorem 5.5 follows from Theorem 5.4. Now we assume that $[\xi, \eta] \neq 0$. First we show that four vector fields $\xi, \eta,[\xi, \eta], F=\operatorname{grad} f$ are linearly independent almost everywhere. Since $\xi$ is a Killing vector field, we get

$$
\begin{aligned}
L_{\xi} f & =L_{\xi}(g(\xi, \eta))=g(\xi,[\xi, \eta]) \\
& =g\left(\xi, \nabla_{\xi} \eta-\nabla_{\eta} \xi\right) \\
& =g(\xi,-\psi \xi+\phi \eta)=0 .
\end{aligned}
$$

Similarly we have $L_{\eta} f=0$. Denoting by $d$ the exterior differentiation we get $L_{\xi} d f=d L_{\xi} f=0$ and $L_{\eta} d f=0$. These relations show

$$
\begin{aligned}
& {[\xi, F]=L_{\xi} F=0,} \\
& {[\eta, F]=L_{\eta} F=0 .}
\end{aligned}
$$

At the same time, we obtain

$$
0=L_{\xi} f=d f(\xi)=g(\xi, F) .
$$

Similarly, $g(\eta, F)=0$. Then we get

$$
\begin{aligned}
& 0=L_{\xi}\left(g\left(\eta, F^{\prime}\right)\right)=g\left(L_{\xi} \eta, F\right)=g([\xi, \eta], F), \\
& 0=L_{\xi}(g(\eta, \eta))=2 g([\xi, \eta], \eta),
\end{aligned}
$$

and $g([\xi, \eta], \xi)=0 . \quad F$ vanishes only on the set of measure zero. Since different two Killing vector fields can not be identical on any open set, $\xi, \eta, F,[\xi, \eta]$ are linearly independent almost everywhere by $\xi \perp F$, $\eta \perp F,[\xi, \eta] \perp \xi,[\xi, \eta] \perp \eta,[\xi, \eta] \perp F$. Assume that $\xi, \eta, F,[\xi, \eta]$ are linearly independent at each point of an open set $U$. $\xi, \eta, F$ belong to 
the 1-nullity distribution $D$ on $U$. Since $D$ is integrable on $U$, we have $[\xi, \eta] \in D$. Therefore the index of 1 -nullity $\mu(x) \geq 4$ on $U$, and consequently $\mu \geq 4$ on $M$. Then we can apply [vii] of $\S 2$, and we get Theorem 5.5.

q.e.d.

COROLLARY 5.6. Let $(M, g)$ be a complete Riemannian manifold of dimension $m ; 3 \leq m \leq 11$. If it admits non-proportional two Sasakian structures, then it is an Einstein space.

Proof. This follows from Theorem 5.5 and [iii] of $\S 2$. q.e.d.

\section{REFERENCES}

[1] K. ABE, A characterization of totally geodesic submanifolds in $S^{n}$ and $C P^{n}$ by an inequality, Tôhoku Math. J., 23 (1971), 139-244.

[2] S. S. ChERN AND N. H. KUIPER, Some theorems on the isometric imbedding of compact Riemann manifolds in Euclidean space, Ann. Math., 56 (1952), 422-430.

[3] Y. H. Clifton aNd R. MaLtz, The $k$-nullity spaces of the curvature operator, Michigan Math. J., 17 (1970), 85-89.

[4] D. Frerus, Totally geodesic foliations, Math. Ann., 188 (1970), 313-316.

[5] D. Ferus, On the completeness of nullity foliations, Michigan Math. J., 18 (1971), 61-64.

[6] A. Gray, Spaces of constancy of curvature operators, Proc. Amer. Math. Soc., 17 (1966), 897-902.

[7] S. IshiHARA AND Y. TASHIRo, On Riemannian manifolds admitting a concircular transformation, Math. J. Okayama Univ., 9 (1959), 19-47.

[8] T. KashiwadA, A note on a Riemannian space with Sasakian 3-structure, Nat. Sci. Rep. Ochanomizu Univ., 22 (1971), 1-2.

[9] Y. Y. Kuo, On almost contact 3-structure, Tôhoku Math. J., 22 (1970), 325-332.

[10] R. Maltz, The nullity spaces of the curvature operator, Cahiers de Topo. et Geom. Diff., 8 (1966), 1-20.

[11] M. Oвата, Certain conditions for a Riemannian manifold to be isometric with a sphere, J. Math. Soc. Japan, 14 (1962), 333-340.

[12] M. OватA, Riemannian manifolds admitting a solution of a certain system of differential equations, Proc. United States-Japan Sem. in Diff. Geom. Kyoto, Japan, 1965, 101-114.

[13] T. Otsuki, Isometric imbedding of Riemann manifolds in a Riemann manifold, J. Math. Soc. Japan, 6 (1954), 221-234.

[14] A. Rosenthal, Riemannian manifolds of constant nullity, Michigan Math. J., 14 (1967), $469-480$.

[15] S. SASAKI, Almost contact manifolds, Lecture notes, I, II, III, Tôhoku Univ.

[16] A. S. SoLodovnikov, The group of projective transformations of a complete analytic Riemannian manifold, Dokl. Acad. Nauk. SSSR., 186 (1969); [Sov. Math. Dokl., 10 (1969), 750-753.].

[17] S. TaChibana and W. N. Yu, On a Riemannian spaces admitting more than one Sasakian structure, Tôhoku Math. J., 22 (1970), 536-540.

[18] S. TANNO, The automorphism groups of almost contact Riemannian manifolds, Tôhoku Math. J., 21 (1969), 21-38.

[19] S. TaNNo, On the isometry groups of Sasakian manifolds, J. Math. Soc. Japan, 22 (1970), 579-590. 
[20] S. TANNo, Killing vectors on contact Riemannian manifolds and fiberings related to the Hopf fibrations, Tôhoku Math. J., 23 (1971), 313-333.

Mathematical Institute

TôHOKU UNIVERSITY,

SENDAI, JAPAN

ADDED IN PROOF: Theorem (*) in the introduction has been completely proved by the present author (: Differential equations of order 3 on Riemannian manifolds [to appear]), and consequently Theorem $(* *)$ is also verified. 